\title{
Platinum Layers Sandwiched between Black Phosphorous and Graphene for Enhanced SPR Sensor Performance
}

\section{Maheswari Pandaram}

Government Arts College, Salem-7

\section{Subanya Santhanakumar}

PSGR Krishnammal College for Women

\section{Ravi Veeran}

Government Arts College Salem-7

Rajesh Karuppaiya Balasundaram ( $\sim$ kbr.nanoptik@gmail.com )

Chikkanna Government Arts College Tiruppur https://orcid.org/0000-0003-0638-2348

\section{Rajan Jha}

Indian Institute of Technology Bhubaneswar

\section{Zbigniew Jaroszewicz}

Institute of Applied Optics, Warsaw

\section{Research Article}

Keywords: Surface Plasmon, Sensitivity, Black Phosphorous, Platinum, Graphene

Posted Date: June 7th, 2021

DOl: https://doi.org/10.21203/rs.3.rs-529493/v1

License: (9) (i) This work is licensed under a Creative Commons Attribution 4.0 International License. Read Full License

Version of Record: A version of this preprint was published at Plasmonics on August 6th, 2021. See the published version at https://doi.org/10.1007/s11468-021-01507-5. 


\title{
Platinum Layers Sandwiched between Black Phosphorous and Graphene for Enhanced SPR Sensor Performance
}

\author{
Maheswari Pandaram ${ }^{1}$, Subanya Santhanakumar ${ }^{2}$, Ravi Veeran ${ }^{1}$, Rajesh Karuppaiya Balasundaram ${ }^{3 *}$, \\ Rajan Jha ${ }^{4}$, Zbigniew Jaroszewicz ${ }^{5}$ \\ ${ }^{1}$ Department of physics, Government Arts College,Salem-7,Tamil Nadu, India. \\ ${ }^{2}$ Department of physics, PSGR Krishnammal College for Women, Coimbatore.Tamil Nadu, India. \\ ${ }^{3}$ Department of Physics, Chikkanna Government Arts College, Tirupur, Tamil Nadu, India. \\ ${ }^{4}$ Nanophotonics and Plasmonics Laboratory, School of Basic Sciences, IIT Bhubaneswar 752050, Odisha, India. \\ ${ }^{5}$ Institute of Applied Optics, Department of Physical Optics, Warsaw, Poland and National Institute of \\ Telecommunications, Warsaw, Poland. \\ Corresponding authors Email id: rajeskb@gmail.com
}

\begin{abstract}
:
Highly sensitivity Surface Plasmon resonance (SPR) sensor consisting of Ag-Pt bimetallic films sandwiched with 2D materials Black Phosphorus (BP) and Graphene over Pt layer in Kretschmann configuration is analyzed theoretically using the Transfer Matrix Method. Numerical results shows that upon suitable optimization of thickness of Ag-Pt and number of layers of BP \& graphene, sensitivity as high as $412^{\circ} /$ RIU can be achieved for p-polarized light of wavelength $633 \mathrm{~nm}$. This performance can be tuned and controlled by changing the number of layers of BP and graphene. Further, the addition of graphene and heterostructures of black phosphorus not only improved the sensitivity of the sensor but keep the FWHM of the resonance curve much smaller than the conventional sensor utilizing $\mathrm{Au}$ as plasmon metal and hence improved the resolution to a significant extent. We expect that this new proposed design will be useful for medical diagnosis, biomolecular detection and chemical examination.
\end{abstract}

Keywords: Surface Plasmon, Sensitivity, Black Phosphorous, Platinum, Graphene

\section{Introduction}

SPR is the one of the most powerful optical techniques used for medical diagnostics, enzyme and gaseous detection, food safety[1-8]. This technique has been widely used for a quick and accurate detection of various physical and biochemical parameters. Most of the SPR based sensor usesKretschmann's attenuated total reflection (ATR)method, in which the p-polarized 
incident light excites a surface plasmon wave(SPW) along the metal-dielectric interfaceon the thin metallic layer deposited on the base of an optical coupling prism [9]. The choice of metallic film is one of the crucial aspect in designing SPR based applications. Usually gold(Au) is considered as the most suitable plasmonic material as it is highly resistive to oxidization, possess better chemical stability and provides higher sensitivity. Silver on the other hand shows better resolution where as it oxidizes easily and possess lessersensitivity compared to Au. The bimetallic configuration of $\mathrm{Au} / \mathrm{Ag}$ utilizes the advantages of both the metals and has been demonstrated in several works. It is noted thatthe usage of bimetallic film though improved the resolution of the sensor its sensitivity seems not much improved [10-13]. Apart from $\mathrm{Au}$ and $\mathrm{Ag}$ more metals have been identified for SPR sensing such as Aluminum(Al),Nickel(Ni),Copper(Cu),Platinum(Pt) and each of these metals has their own advantagesand disadvantages [14-16]. Recently, Platinum has been identified as a potential SPR active metal owing to its strong dependence of reflection coefficient on wavelength in the visible region of the spectrum, high reflecting property, inert, chemically stable with high melting point, and prolonged stability [17-19]. Shukla et al. theoretically analyzed the performance of the SPR sensor with Platinum layer coated on optical fiber. They observed that the sensitivity of the sensor enhances linearly with the increase in the refractive index of the sensing medium for all thickness of platinum layer and for a given refractive index of the sensing medium [20].Recently,Black Phosphorus (BP) is identified as one the of potential 2D material [21] which gains rapid attention due to its widely tunable and direct bad gap, remarkable electrical and optical properties and higher carrier mobility [22-25]. The BP also provides attractive physical, chemical and mechanical anisotropic properties which makes it a suitable candidate for high performance potential and chemical applications [26]. Srivastava et.al reported that implementation of double layer BP enhanced the sensitivity by 35\%[27].Recently, Graphene on the other hand exhibits remarkable properties such ashigh charge carrier mobility which inducestrong coupling at the interface between metal-graphene films [28].Wu et al. reported utilizing a graphene enhance the sensitivity by $25 \%$ compared with conventional goldbased SPR sensor[29]. Recently many studies on the effect of graphene on SPR sensorsensitivity enhancement is attempted from numerical aspects [30-31].Furthermore, the graphene is impermeable to gases such as oxygen and helium because electron density of hexagonal rings is enough to prevent atoms and molecules from passing through the ring structure [32]. Hence 
graphene can be used as protective layer against oxidation of metals which is also found to improve the sensitivity of the sensor to a great extend [33]. Recent, SPR biosensor based on Blue P-TMDC-graphene hetero structure [34], graphene- $\mathrm{BaTiO}_{3}$ nanosheets[35] have also been proposed.

In this paper, we have designed a new sensor configuration composed of Ag-Pt bimetallic films sandwiched with 2D materials Black Phosphorous(BP) and graphene over Pt layer. Here, we report that ultra-high sensitivity of the SPR sensor can be realized upon suitable optimization of thickness of metal layers and no. layers of BP and graphene. Here it is noted that the proposed sensing configuration not only enhancessensitivity but still keep the FWHM of the resonance curve much smaller than the conventional sensor utilizing $\mathrm{Au}$ as plasmonic material and hence improved the resolution to a greater extend. Moreover, the top surface graphene coat not only protect the metal surface but improved the biomolecular absorption through high surface to volume ratio and Pi conjugation structure.

\section{Theory}

The structure of the proposed SPR biosensor is given in Fig.1 which consist ofmultilayer (six layers) structure. Keeping in mind the widely used prism required for momentum match, the first layer is of BK7 prism. The second layer is aoptimized thickness of $40 \mathrm{~nm}$ thickness of silverwhich is deposited on the base of the coupling prism. The third and fourth layers are BP and optimized thickness of $15 \mathrm{~nm}$ thickness of platinum. The fifth layer is consider as graphene followed by sensing layer.TM-polarized light, wavelength of $633 \mathrm{~nm}$ is used at one face of the prism and is assumed to be collected from the other face with proper optics. The dispersion of prism is considered as [36]

$n_{B K 7}=\left(\frac{1.03961212 \lambda^{2}}{\lambda^{2}-0.00600069867}+\frac{0.231792344 \lambda^{2}}{\lambda^{2}-0.0200179144}+\frac{1.03961212 \lambda^{2}}{\lambda^{2}-103.560653}+1\right)^{1 / 2}$

Where $\lambda$ is the wavelength of incident light in $\mu m$.

The wavelength dependence dielectric constant of silver is calculated using Drude Lorentz formula as given by

$$
\varepsilon_{m}(\lambda)=\varepsilon_{m r}+\varepsilon_{m i}=1-\frac{\lambda^{2} \lambda_{c}}{\lambda_{p}^{2}\left(\lambda_{c}+i \lambda\right)}
$$


Where, $\lambda_{p}=1.4541 \times 10^{-7} \mathrm{~m}$ and $\lambda_{c}=1.7614 \times 10^{-5}$ m.here, $\lambda_{p}$ and $\lambda_{c}$ be the plasma and collision wavelength of silver $(\mathrm{Ag})$ respectively[33]. The third layer is BP whose refractive index is $\mathrm{n}_{3}=$ $3.5+0.01 \mathrm{i}$ at $\lambda=633 \mathrm{~nm}$ and the thickness of BP is calculated as $\mathrm{d}_{3}=\mathrm{M} \times 0.53 \mathrm{~nm}$. where, number of Black Phosphorus(BP) layer is indicated by M[37]. Fourth layer is platinum (Pt) layer and its dielectric constant is calculated according to the Drude model as given in equation (3), with $\lambda_{p}=$ $2.415 \times 10^{-7} \mathrm{~m}$ and $\lambda_{c}=1.795 \times 10^{-5} \mathrm{~m}$ [38]. The fifth layer is made of graphene and its refractive index $\left(\mathrm{n}_{5}\right)$ is given as

$$
n_{5}=3.0+i \frac{C_{1}}{3} \lambda
$$

Where the constant $\mathrm{C}_{1} \approx 5.446 \mu \mathrm{m}^{-1}[39]$.

The sixth layer is the sensing medium whose refractive index is assumed to change from 1.33 to 1.335 as if the suitable for biomolecules observation. We have used matrix for N-layer model in order to obtain the intensity of reflected light (reflectance), since the matrix method is very accurateand can be applied to a system containing any number of layers. $M$ is characteristic matrix of the combined structure and for p-polarized light it is given by

$$
M=\prod_{k=2}^{N-1} M_{k}=\left[\begin{array}{ll}
M_{11} & M_{12} \\
M_{21} & M_{22}
\end{array}\right] \text { With }
$$

$$
M_{k}=\left[\begin{array}{cc}
\cos \beta_{k} & \frac{-i \sin \beta_{k}}{q_{k}} \\
-i q_{k} \sin \beta_{k} & \cos \beta_{k}
\end{array}\right]
$$

where

$$
q_{k}=\left(\frac{\mu_{k}}{\varepsilon_{k}}\right)^{1 / 2} \cos \theta_{k}=\frac{\left(\varepsilon_{k}-n_{1}^{2} \sin ^{2} \theta_{1}\right)^{1 / 2}}{\varepsilon_{k}}
$$

and

$$
\beta_{k}=\frac{2 \pi}{\lambda} n_{k} \cos \theta_{k}\left(z_{k}-z_{k-1}\right)=\frac{2 \pi d_{k}}{\lambda}\left(\varepsilon_{k}-n_{1}^{2} \sin ^{2} \theta_{1}\right)^{1 / 2}
$$


where $\theta$ and $\lambda$ represents angle and wavelength of the incident light. Here,present model, there are six layers, hence $\mathrm{N}=6$. Thus transfer matrices are calculated in accordance with Eq. (6). The corresponding amplitude reflection coefficient $\left(r_{p}\right)$ will be

$$
r_{p}=\frac{\left(M_{11}+M_{12} q_{N}\right) q_{1}-\left(M_{21}+M_{22} q_{N}\right)}{\left(M_{11}+M_{12} q_{N}\right) q_{1}+\left(M_{21}+M_{22} q_{N}\right)}
$$

In the above equation, $\mathrm{q}_{1}$ and $\mathrm{q}_{\mathrm{N}}$ corresponds to the BK7 substrate and the sensing layer respectively, and finally the reflectivity $\left(\mathrm{R}_{\mathrm{p}}\right)$ can be given as

$\mathrm{R}_{\mathrm{p}}=\left|r_{p}\right|^{2}$

Sensitivity of the SPR biosensor is measured as the small change in the refractive index $\left(\Delta n_{s}\right)$ of the analyte with the change in resonance condition in the reflectance curve $\left(\Delta \theta_{\text {res }}\right)$; therefore the sensitivity is given by,

$$
S=\frac{\Delta \theta_{\text {res }}}{\Delta n_{s}}
$$

The other prominance parameters of the SPR sensors are Quality Factor (Q), and Signal to Noise ratio(SNR) these parameters should be high for the good sensors.SNR isdefined as the ratio of resonance angle shift $\left(\Delta \theta_{\text {res }}\right)$ and the full widthat half maxima (FWHM) $\left(\Delta \theta_{0.5}\right)$ of the reflectance curve,

$$
S N R=\frac{\Delta \theta_{r e s}}{\Delta \theta_{0.5}}
$$

The quality factor $(\mathrm{Q})$ is given by,

$$
Q=\frac{S}{\Delta \theta_{0.5}}
$$

where, $\mathrm{S}(\theta)$ - Sensitivity of the SPR biosensor 
$\Delta \theta_{0.5}-$ FWHM of the SPR curve

\section{Results and Discussions}

Here, we have numerically simulated and analyzed the performance of the proposed configuration using the transfer matrix method. Here, the conditionof minimum reflectance $\left(\mathrm{R}_{\min }\right)$ close to zero ensures coupling of maximum energy of incident TM polarized light with the surface plasmon and it is necessary for the design of any SPR sensor to have improvised sensitivity and resolution[40-42]. In order to ensure such a condition here, fixed one such possible configuration with thickness of $\mathrm{Ag}$ as $40 \mathrm{~nm}$ and Pt as $15 \mathrm{~nm}$. Fig.2(a) shows the reflectance curve plotted corresponding to the change in R.I $\left(\Delta n_{s}\right)=0.005$ in the absence of BP and graphene $(\mathrm{M}=0$ and $\mathrm{L}=0)$. As can been clearly seen from figure that the shift in the resonance angle is measured as $\Delta \theta=1.21^{\circ}$ and the corresponding sensitivity as per eqn.9 is calculated as $240.76 \mathrm{deg} /$ RIU.Fig.2(b\&c)shows that upon introducing a single layer of either BP orgraphene ( $\mathrm{M}=1$ and $\mathrm{L}=0$ or $\mathrm{M}=0$ and $\mathrm{L}=1$ ) generates the shift in resonance angle as $\Delta \theta=1.26^{\circ}$ and the corresponding sensitivity as per eqn.9 is $252.23 \mathrm{deg} / \mathrm{RIU}$. Hence it is to be noted that the addition of either the mono layer of graphene or BP is found to have same effect on the sensitivity of the sensor. However, it is further noted from Fig. 2(d) that for the proposed sensor configuration with the inclusion of both mono layer of $\mathrm{BP}$ and graphene $(\mathrm{M}=1$ and $\mathrm{L}=1)$, the shift in the resonance curve is found to be significantly improved as $\Delta \theta=1.38^{\circ}$ due to the different value of dielectric constant of the materials which fulfils the resonance condition at different angle. Thecorresponding sensitivity increases as high as 275.15deg/RIU. As, the sensitivity enhancement depends on the absorption of incident light in the different layers, the proposed configuration with single layers of BP and graphene shows better absorption and hence exhibitsmuch improvement in the sensitivity compared with traditional structure [43].

Fig. 3(a) shows the shift in dip of SPR curve corresponding to the change in R.I of the sensing medium in the range of 1.330 to 1.350 . It is noted that, without $\mathrm{BP}$ and graphene $(\mathrm{M}=0$ and $\mathrm{L}=0$ ) the shift in the dip varies from $76.18^{\circ}$ to $81^{\circ}$ whereas it increased from $76.75^{\circ}$ to $82.4^{\circ}$ for the configuration with inclusion of mono layer of $\mathrm{BP}(\mathrm{M}=1$ and $\mathrm{L}=0)$ which is due to strong dispersion of $\mathrm{BP}$ around incident wavelength. It is also noted that further increases in $\Delta \theta$ around 
$76.96^{\circ}$ to $82.4^{\circ}$ is obtained for the configuration with mono layer of graphene $(\mathrm{M}=0$ and $\mathrm{L}=1)$ which shows that better absorption property of graphene over BP. However for theconfiguration with theinclusion of both $\mathrm{BP}$ and graphene $(\mathrm{M}=1$ and $\mathrm{L}=1)$ layers, the shift in resonance curve dipmuch improved from $78.65^{\circ}$ to $85.58^{\circ}$ which is due to the combined effect of both the layers. Fig 3(b) shows the variation of sensitivity of the proposed sensor with respect to the refractive index of the sensing medium by keeping the other parameter same as before. We observed that for the configuration without BP and graphene layers, the sensitivity increased from 206.29 to 332.48deg/RIU(M=0 and $\mathrm{L}=0)$. However the inclusion of $\mathrm{BP}(\mathrm{M}=1$ and $\mathrm{L}=0)$, the sensitivity is found to varies from 217.83 to $378.34 \mathrm{deg} / \mathrm{RIU}$, where as an addition of monolayer of graphene without $\mathrm{BP}(\mathrm{M}=0$ and $\mathrm{L}=1)$, enhance the sensitivity from 229.29 to $389.8 \mathrm{deg} / \mathrm{RIU}$. It is also noted that for the configuration considered withinclusion of both monolayer of BP and graphene $(\mathrm{M}=1$ and $\mathrm{L}=1)$, further improved the sensitivity from 252.2 to $435.6 \mathrm{deg} / \mathrm{RIU}$ which is due to the combined effect of both the $2 \mathrm{D}$ materials as the dispersion variation is different for both BP and graphene which results in a modified effective index around the wavelength of operation. Here, we concluded that the addition of BP and graphene layers can significantly improve the sensitivity of the proposed biosensor compared with the conventional gold based SPR structure[43].

Further, we optimized the no. of graphene and BP layers required to achieve best performance of the proposed sensor. Fig. 4(a) shows the reflection spectra versus angle of incidence obtained for a mono layer of graphene with increasing number of Black phosphorus(BP) layers for $\mathrm{Ag}=40 \mathrm{~nm}$ and $\mathrm{Pt}=15 \mathrm{~nm}$. It is noted from the figure 4(a) that increasing number of BP layer largely shifts the reflectance dip as the resonance condition is satisfied at higher angleand hence improved the sensitivity, which is due to higher mobility of charge carriers and higher absorption efficiency of BP[44]. We also observed that similar shift in resonance curve is also noted forthe addition of graphene layers as shown in fig. 4(b) and found to be larger than the addition of BP layers owing to the large real part of dielectric constant of graphene.

It is also noted from both the figures $4(\mathrm{a} \& \mathrm{~b})$ that increasing either the no.of BP layers and graphene layers not only generates larger shift in resonance dip but also makes the resonance curve wider and shifts the $\mathrm{R}_{\min }$ to higher values. Such an increase in FWHM of the spectral curve is due to the decrease of propagation velocity of SP waves in 2D materials which 
results in damping[45].The increase in $\mathrm{R}_{\min }$ is due to the saturation in absorption of incident light energy and the increase of electron loss[46-48] It is also noted that widening of resonance curve and increase in $\mathrm{R}_{\min }$ is higher for graphene due to its large imaginary part of dielectric constant when compared to BP. This meansarbitrarily increasing either BP or graphene layers makes the SPR curve broader and its more difficult to measure near the resonance angle, thereby affecting the accuracy of the measurement. Thus, we can conclude that one cannot arbitrarly increase the number of BP layers and graphene layers for the optimization of sensitivity.

Based on the above analysis, to optimize the number of BP and graphene layers, we calculated the sensitivity for BP and graphene layers. Fig.5 shows the minimum reflectance $\left(R_{\min }\right)$ and sensitivity corresponding to number of graphene layer with a mono layer of $\mathrm{BP}$ with $\mathrm{Ag}=40 \mathrm{~nm}$ and $\mathrm{Pt}=15 \mathrm{~nm}$. It is clearly observed that the sensitivity of the proposed configuration improved with increasing number of graphene layers till 4 and then sensitivity starts to decrease. This is because of increasing of minimum reflectance $\left(R_{\min }\right)$ from $4^{\text {th }}$ layers onwards as it reduce the absorption of light ie., optical energy losses occurred due to increasing number of graphene layers.Hence for the proposed configuration we optimized the no. of graphene layers as 4 for the best performance. The $\mathrm{R}_{\min }$ and sensitivity obtained for theoptimized graphene layer $(\mathrm{L}=4)$ are 0.0336 and $401 \mathrm{deg} / \mathrm{RIU}$ respectively. Fig.6 shows the variation of $\mathrm{R}_{\min } \&$ sensitivity versus number of BP layer with optimized no.layer of graphene $(\mathrm{L}=4)$. From, Fig.6, it is noted that the sensitivity of the proposed sensor increase with increasing the BP layer till the $2^{\text {nd }}$ layers and it starts decreasing sharply. This suggests, we just need to choose $M=2$ for getting a maximum sensitivity for sensing applications. It is observed that after optimization of $\mathrm{BP}$ and graphene layer $(\mathrm{M}=2$ and $\mathrm{L}=4)$ the maximum sensitivity of the proposed biosensor is about $412 \mathrm{deg} /$ RIU and still keep the FWHM value as $5.731^{\circ}$. The signal to noise ratio(SNR) and quality factor(Q) are calculated using eqns.(10-11), and are found to be as $0.36 \mathrm{deg}^{-1} \& 71.88 \mathrm{RIU}^{-1}$ respectively.Further, to have a better insight into the electric field distribution at different layer in the proposed multilayered system. Fig.7, shows the normalized transverse electric field distribution as a function of distance normal to the interface. It is noted from the figurethat the Aglayer enhances the electric field and shows a peak at Ag-BP interface. The field intensity falls in the BP layers and further gets much enhanced in the platinum (Pt) layer which shows the excitation of SP's at this interface. It is noted that addition of graphene layer further increases the field intensity which reaches its maximum at the graphene-sensing medium interface and 
decays exponentially in the sensing medium. This enhanced probing field close to the graphene layer with long probing depth is highly sensitive to bio molecular interaction throughout the penetrating depth.Such an enhanced probing evanescent field increases the interaction volume and hence maximize the sensitivity of the sensor[49-50].

Finally, we concluded that the proposed sensor with the configuration of 40nm thickness of $\mathrm{Ag}$, with two layer of $\mathrm{BP}, 10 \mathrm{~nm}$ of platinum $(\mathrm{Pt})$ and four layers of graphene can enhance the sensitivity as high as $412 \mathrm{deg} / \mathrm{RIU}$ with FWHM of $5.731^{\circ}$. The signal to noise ratio and quality factor values are noted as $0.36 \mathrm{deg}^{-1} \& 71.88 \mathrm{RIU}^{-1}$ respectively. The FWHM obtained here is nearly close to the Au-Ag bimetallic film based SPR sensor however the sensitivity achieved is found to be much higher[51]. Some of the relevant works and its sensitivity has been compared in table1. We expect on the basis of the enhanced performance of sensitivity and lower FWHM of the SPR curve, the proposed sensor will have better performance in the chemical and biological applications.

\section{Conclusion:}

In this paper, we theoretically proposed an SPR sensorconfiguration sandwichingBlack Phosphorus(BP) layers between silver and graphene coated platinum in the Kretschmann set up.We observed that, by properly optimizing the structure of the sensor, sensitivity can beenhanced as high as $412^{\circ} /$ RIU and can still keep the FWHM of the resonance curve small as 5.731deg.We also noted that the sensitivity can be tuned and controlled by changing the number of layers of BP and graphene. We expect that such a proposed configuration exhibiting enhancedsensitivity and lower FWHM would make our configuration to have more applications in food safety, chemical examination, and biological detection.

\section{Funding:}

Not Applicable

\section{Conflict of interest/competing interest:}

No potential conflict of interest was reported by the authors.

\section{Availability of data and material:}


Data and material will be made available on reasonable request.

\section{Code availability:}

Code will be made available on reasonable request.

Author's contributions:

Maheswari Pandaram - Conceptulization, data curation, Original draft preparation

Subanya Santhanakumar - Software, Validation

Ravi Veeran - Reviewing and Editing

Rajesh Karuppaiya Balasundaram - Supervision, Writing - Reviewing and Editing

Rajan Jha

Zbigniew Jaroszewicz

Ethics approval:

Not applicable.

\section{Consent of participate:}

Not applicable .

\section{Consent of publication:}

Not applicable .
- Reviewing and Editing

- Reviewing and Editing

\section{References}

[1].Liedberg B, Nylander C, Sundstrom I (2005) Surface plasmon resonance for gas detection andiosensing. Sensors and Actuators B 4:299-304.

[2]. Jorgenson RC, Yee SS(1993) A fiber-optic chemical sensor based on surface plasmon resonance. Sensors and Actuators B 12: 213-220. 
[3]. Wang TJ, Tu CW, Liu FK, Chen HL (2004) Surface Plasmon Resonance waveguide biosensor by bipolarization wavelength interrogation. IEEE Photonics Technology Letters 16:1715-1717.

[4]. Srivastava T, Purkayastha A, Jha R (2016)Graphene based surface plasmon resonance gas sensor for terahertz. Optical and Quantum Electronics 48(6) : 334.

[5]. Choi SH, Kim YL, Byun KM (2011) Graphene-on-silver substrates for sensitive surface plasmonresonanceimagingbiosensors. Optics Express 19: 458-466.

[6].Nayak JK, Jha R (2018) Graphene- oxide coated Ag-island based Inline LSPR fiber sensor. Photonics Technology Letters 30(19) : 1667-1670.

[7].Nayak JK, Parhi P, Jha R (2016) Experimental and Theoretical studies on Localized surface plasmon resonance based Fiber optic Sensor using Graphene oxide coated silver nanoparticles. Journal of Physics D: Applied Physics 49 : 285101.

[8]. Srivastava T, Das R, Jha R (2011) Highly accurate and sensitive surface plasmon resonance sensorbasedonchannelphotoniccrystalwaveguides. SensorsandActuators B 57:246-252.

[9]. Kretschmann E, Reather H (1968) Radiative decay of non-radiative surface plasmons excited by light. Zeitschrift fur Nature forschung 23:2135-21.

[10]. Maharana PK, Bhardwaj S, Jha R (2013) Electric field enhancement in surface plasmon resonance bimettalic configuration based on chalcogenide prism. Journal of Applied Physics 114 : 014304-4.

[11]. Wang Y, Meng S, Liang Y, Li L, Peng W(2013) Fiber optic surface plasmon resonance sensor with multi-alternating metal layers for biological measurement. Photonic Sensors 3: 202-207.

[12]. Jha R, Sharma AK (2009) Chalcogenide glass prism based SPR sensor with Ag-Au bimetallic nanoparticle alloy in infrared wavelength region. Journal of Optics A: Pure and Applied Optics 11:045502.

[13]. Shalabney A, Abdulhalim I (2011)Sensitivity-enhancement methods for surface plasmon sensors.Laser Photonics Rev 5:571-606.

[14]. Vibisha GA, Nayak JK, Maheswari P, Priyadharsini N, Nisha A, Jaroszewicz Z, Jha $R(2020)$ Sensitivity enhancement of surface plasmon resonance sensor using hybrid configuration of 2D materials over bimetallic layer of Cu-Ni. Optics Comm. 463:125337. 
[15]. Oliveira LC, Moreira CS, Neff H, Lima AM(2016) Optical properties and instumental performance of thin noble metal $(\mathrm{Cu}, \mathrm{Au}, \mathrm{Ag})$ films near the surface plasmon resonance. Procedia Engineering 168:834-837.

[16]. Maurya JB, Prajapati YK (2016) A comparative study of different metal and prism in the surface plasmon resonance biosensor having $\mathrm{MoS}_{2}$-graphene. Opt Quant Electron 48(5):1-12.

[17]. Velichkina LM,Pestryakov AN,Vosmerikov AV,Tuzovskaya IV, Bogdanchikova NE, Avalos M,Farias M,Tiznado H (2008) Catalytic activity in hydrocarbon conversion of pentasil containing platinum, nickel, iron, or zinc nanoparticles. Petroleum Chemistry 48:201 -205.

[18]. L.M.Velichkina, A.N.Pestryakov, A.V.Vosmerikov, I.V.Tuzovskaya,

N.E.Bogdanchikova, M.Avalos,M.Farias,H.Tiznado,Catalytic activity in the hydrocarbon conversion of system containing platinum, nickel, iron, or zinc nanoparticles. Petroleum Chemistry 48 (2008) 355-359.

[19]. Chen A,Holt-Hindle P(2010) Platinum based nanostructured materials, Synthesis, properties, and applications. Chemical reviews 110 : 3767-3804.

[20]. Shukla S, Rani M, Sharma MK, Sajal V(2015) Sensitivity enhancement of Surface plasmon resonance based fiber optic sensor utilizing platinum layer. Optik126 : 46364639.

[21]. Churchill HO, Jarilloherrero $\mathrm{P}(2014)$ Two-dimensional crystals: phosphorus joins the family. Nat Nanotechnol 9(5) : 330-331.

[22]. Li L, Yu Y, Ye GL, Ge Q, Ou X, Wu H, Feng D, Chen XH, Zhang Y(2014) Black phosphorus field-effect transistors. Nat Nanotechnol 9 (5) : 372-377.

[23]. Qiao J, Kong X, Hu ZX, Yang F, Ji W(2014) High-mobility transport anisotropy and linear dichroism in few-layerblack phosphorus.Nat Commun $5: 4475$.

[24]. Rodin AS, Carvalho A, Castro Neto AH(2014) Strain-induced gap modification in black phosphorus. Phys Rev Lett 112 (17) : 176801-176801.

[25]. Wang X, Jones AM, Seyler KL, Tran V, Jia Y, Zhao H, Wang H, Yang L, Xu X, Xia F(2014) Highly anisotropic and robust excitons in monolayer black phosphorus. Nat Nanotechnol 10 (6) : 517-21. 
[26]. Nannan Mao et.al (2016) optical anisotropy of BP in the visible regime. J Am Chem Soc 138(1) : 300-305.

[27]. Triranjita Srivastava, Rajan Jha (2018) Black Phosphorus: A new platform for gaseous sensing based on Surface Plasmon Resonance. IEEE 99 : 1-1.

[28]. Elias DC, Gorbachev RV, Mayorov AS, Morozov SV, Zhukov AA, Blake P, Ponomarenko LA, Grigorieva IV, Novoselov KS, Guinea F, Geim AK (2011) Dirac cones reshaped by interaction effects in suspended graphene. Nat Phys 7: 701-704.

[29]. Wu L, Chu HS, Koh WS, Li EP (2010) Highly sensitive graphene biosensors based on surface plasmon resonance Opt Express 18 : 14395-14400.

[30]. Choi SH, Kim YL, Byun KM (2011) Graphene-on-silver substrates for sensitive surface plasmon resonance imaging biosensors. Opt Express 19:458-466.

[31]. Nayak JK, Maharana PK, Jha R (2017) Dielectric over-layer assisted graphene, its oxide and MoS2 based fibre optic sensor with high field enhancement. Journal of Physics D: Applied Physics 50 : 405112.

[32]. Jiang DE, Cooper VR, Dai S (2009) Porous graphene as the ultimate membrane for gas separation. Nano Letter 9(12) : 4019-4024.

[33]. Bahar Meshginqalam, Barvestani J (2018) Performance enhancement of SPR Biosensor based onPhosphorene and Transition Metal Dichalcogenides for Sensing DNA Hybridization. IEEE sensors Journal 18 : 7537-7543.

[34]. Lei Han, Zhimin Hu, Jianxing Pan, Tianye Huang, Dapeng Luo (2020) High Sensitivity Goos-Hanchen shifts sensor based on Blue P-TMDC-Graphene Heterostructure. Sensors 20(12) : 3605 .

[35]. Amrindra Pal, Ankit Jha (2021) A Theoretical Analysis on sensitivity improvement of an SPR Refractive index sensor with graphene and BaTiO nanosheets. Optik 231 : 166378.

[36]. Lin Z, Jiang L, Wu L, Guo J, Dai X, Xiang Y, Fan D (2016) Tuning and sensitivity enhancement of surface plasmon resonance biosensor with graphene covered Au-MoS2Au films. IEEE Photonics J 8(6) : 4803308.

[37]. Leiming Wu, Jun Guo, Qingkai Wang, Shunbin Lu, Xiaoyu Dai, Yuanjiang Xiang, Dianyuan FanSZU (2017) Sensitivity enhancement by using few-layer blackphosphorus- 
graphene/TMDCs heterostructure in surface plasmon resonance biochemical sensor. Sensors and Actuators B 249 : 542-548.

[38]. Ordal MA, Bell RJ, Alexander Jr RW, Long LL, Querry MR (1985) Optical properties of fourteen metals in the infrared and far infrared: $\mathrm{Al}, \mathrm{Co}, \mathrm{Cu}, \mathrm{Au}, \mathrm{Fe}, \mathrm{Pb}, \mathrm{Mo}$, Ni, Pd, Pt, Ag, Ti, V, and W. Applied Optics 24 : 4493-4499.

[39]. Nayak JK, Jha R (2017) Numerical simulation on the performance analysis of a graphene coated optical fiber plasmonic sensor at anti-crossing. Applied Optics 56(12) : 3510-3517.

[40]. Tiwari K, Sharma SC, Hozhabri N (2015) High performance surface plasmon sensors: Simulations and measurements. J Appl Phys 118(9) : 093105.

[41]. Maharana PK, Padhy P, Jha R (2013)On the field enhancement and performance of an ultra stable SPR biosensor based on graphene. IEEE Photonics Technol Lett25 (22): 2156-2159.

[42]. Srivastava, Jha R (2020) Plexcitonic nose based on an organic semiconductor. Applied Physics Letters 117 (9) : 093301.

[43]. Mishra AK, Mishra SK, Verma RK (2015)An SPR-based sensor with an extremely largedynamic range of refractive index measurements in the visible region. J Phys D: ApplPhys 48(43) : 435502.

[44]. Lei Han, Zhanxing Chen, Tianye Huang, Huafeng Ding, Chuan Wu (2020) Sensitivity Enhancement of Ag-ITO-TMDCs-Graphene Nanostructure Based on Surface Plasmon Resonance Biosensors. Plasmonics 15 : 693-701.

[45]. Maharana PK, Srivastava T, Jha R (2014) Low index dielectric mediated surface plasmon resonance sensor based on graphene for near infrared measurements. J Phys D: Appl Phys 47 : 385102.

[46]. Zeng S, Hu S, Xia J, Anderson T, Dinh XQ, Meng XM, Coquet P, Yong KT (2015), Graphene -MoS2 hybrid nanostructures enhanced surface plasmon resonance biosensors, Sens. Actuators B Chem 207 : 801-810.

[47]. Zhao X, Huang T, Ping PS, Wu, X, Huang P, Pan J, Wu Y, Cheng Z (2018) Sensitivity enhancement in surface plasmon resonance biochemical sensor based on transition metal dichalcogenides/graphene heterostructure, Sensors 18(7) : 2056. 
[48]. Ouyang Q, Zeng S, Dinh XQ, Coquet P, Youg KT (2016) Sensitivity enhancement of MoS2 nanosheet based surface plasmon resonance biosensor. Procedia Eng 140 : 134139.

[49]. Shalabney A, Abdulhalim I (2010) Electromagnetic fields distribution in multilayer thin film structures and the origin of sensitivity enhancement in surface plasmon resonance sensors. Sen. Actuators A 159 :24-32.

[50]. Maurya JB, Prajapati YK, Singh V, Saini JP (2015) Sensitivity enhancement of surface plasmon resonance sensor based on graphene-MoS2 hybrid structure with $\mathrm{TiO}_{2}-\mathrm{SiO}_{2}$ composite layer. Applied Physics A 121 : 525-533.

[51]. Wang M, Huo Y, Jiang Z, Zhang C, Yang C, Ning T, Liu Z, Li C, Zhanga W, Mana B (2017) Theoretical design of a surface plasmon resonance sensor with high sensitivity and high resolution based on graphene-WS2 hybrid nanostructures and $\mathrm{Au}-\mathrm{Ag}$ bimetallic film. RSC Adv 7 : 47177.

[52]. Maurya JB, Prajapati YK, Singh V, Saini JP, Tripathi R (2016) Improved performance of the surface plasmon resonance biosensor based on graphene or $\mathrm{MoS}_{2}$ using silicon. Opt Commun 359 : 426-434.

[53]. Rahman M, Anower M, Hasan M, Hossain M, Haque M (2017) Design and numerical analysis of highly sensitive $\mathrm{Au}-\mathrm{MoS}_{2}$-graphene based hybrid surface plasmon resonance biosensor. Opt Commun $396: 36-43$.

[54]. Sarika Pal, Alka verma, Raikwar S, Prajapati YK, Saini JP (2018) Detection of DNA hybridization using graphene- coated black phosphorus surface plasmon resonance sensor. Applied Physics A 124(5) :1-11.

[55]. Mohamed Alagdar, BedirYousif.Nehal, Areed Mahmound Elzalabani F(2019) Improved the quality factor and sensitivity of a surface plasmon resonance sensor with transition metal dichalcogenide 2D nanomaterials. Journal of Nanoparticle Research 22 : 189.

[56]. Rajeev Kumar, Sarika Pal, Alka Verma, Prajapati YK, . Saini JP (2020) Effect of silicon on sensitivity of SPR biosensor using hybrid nanostrucutre of black phosphorus and MXene. Super lattices and nanostrucutres $145: 106591$ 


\section{Table 1:}

\section{Comparison among proposed biosensor with other existing biosensor}

\begin{tabular}{|l|l|l|l|}
\hline S. NO & Configuration & $\begin{array}{l}\text { Sensitivity } \\
(\mathbf{d e g} / \text { RIU })\end{array}$ & Reference \\
\hline 1. & $\begin{array}{l}\text { Prism/Au/graphene/affinity } \\
\text { layer/sensing layer }\end{array}$ & 27.29 & {$[53]$} \\
\hline 2. & $\begin{array}{l}\text { Prism/Au/graphene/MoS } / \text { PBS } \\
\text { solution }\end{array}$ & 87.8 & {$[54]$} \\
\hline 3. & $\begin{array}{l}\text { Prism/Few layer BP } \\
\text { film/graphene/PBS solution }\end{array}$ & 125 & {$[55]$} \\
\hline 4. & $\begin{array}{l}\text { Prism/Chromium/Au/BP/2Dmaterial } \\
\text { Prism/Air } \\
\text { gap/Ag/ITO/MoS2/graphene }\end{array}$ & 187 & {$[34]$} \\
\hline 5. & $\begin{array}{l}\text { Prism/Ag/WS2/Ni/graphene/sensing } \\
\text { medium }\end{array}$ & 243.31 & {$[56]$} \\
\hline 6. & Prism/Ag/Si/BP/Mxene & 264 & {$[28]$} \\
\hline 8. & Prism/Ag/BP/WSe2 & 279 & {$[38]$} \\
\hline 9. & Prism-air-WS2-Al-WS2-graphene & 315.52 & {$[48]$} \\
\hline 10. & $\begin{array}{l}\text { Prism/Ag/BP/Pt/graphene/sensing } \\
\text { medium }\end{array}$ & 412 & $\begin{array}{l}\text { Proposed } \\
\text { work }\end{array}$ \\
\hline
\end{tabular}


Figures

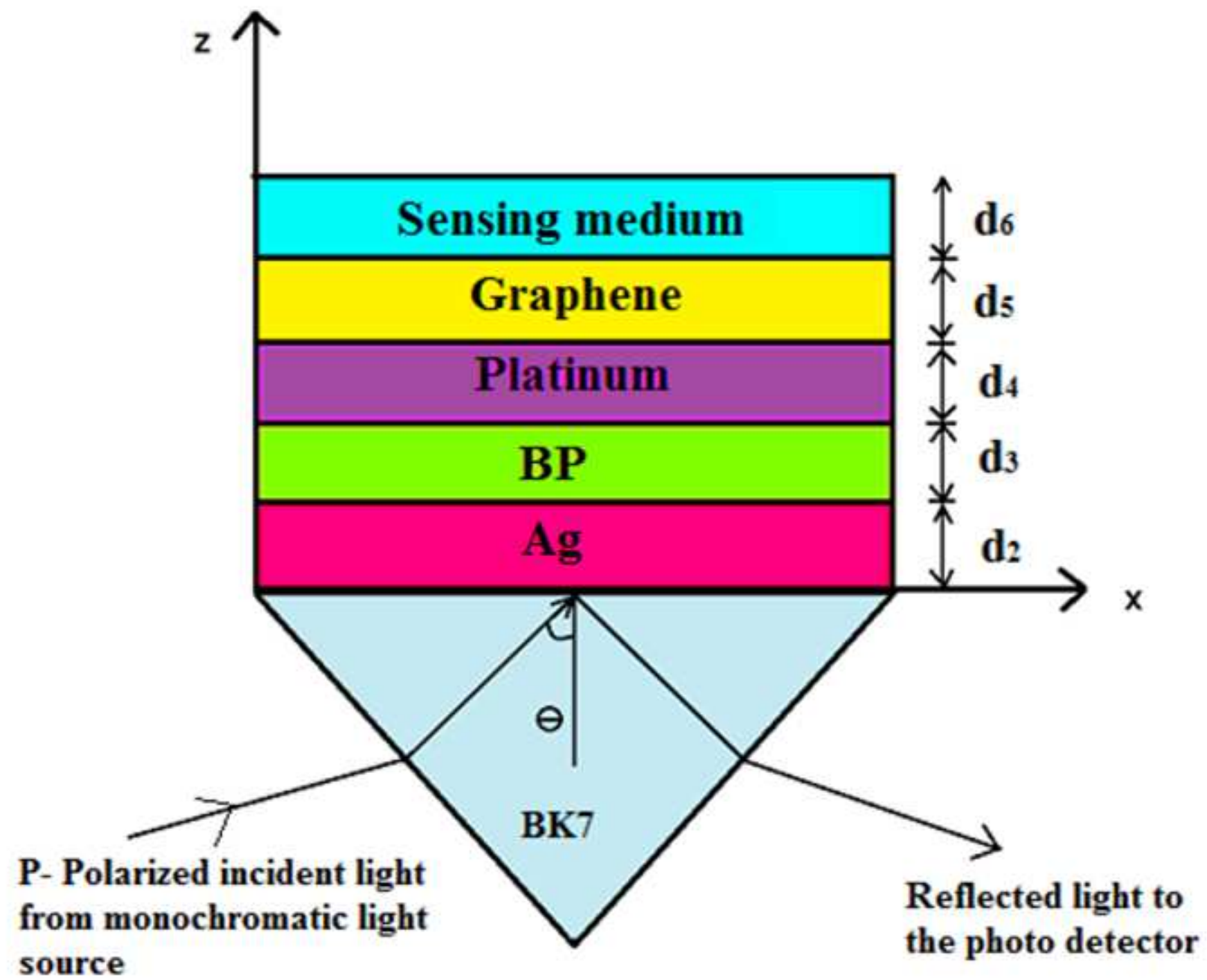

Figure 1

Schematic diagram of the proposed SPR biosensor 

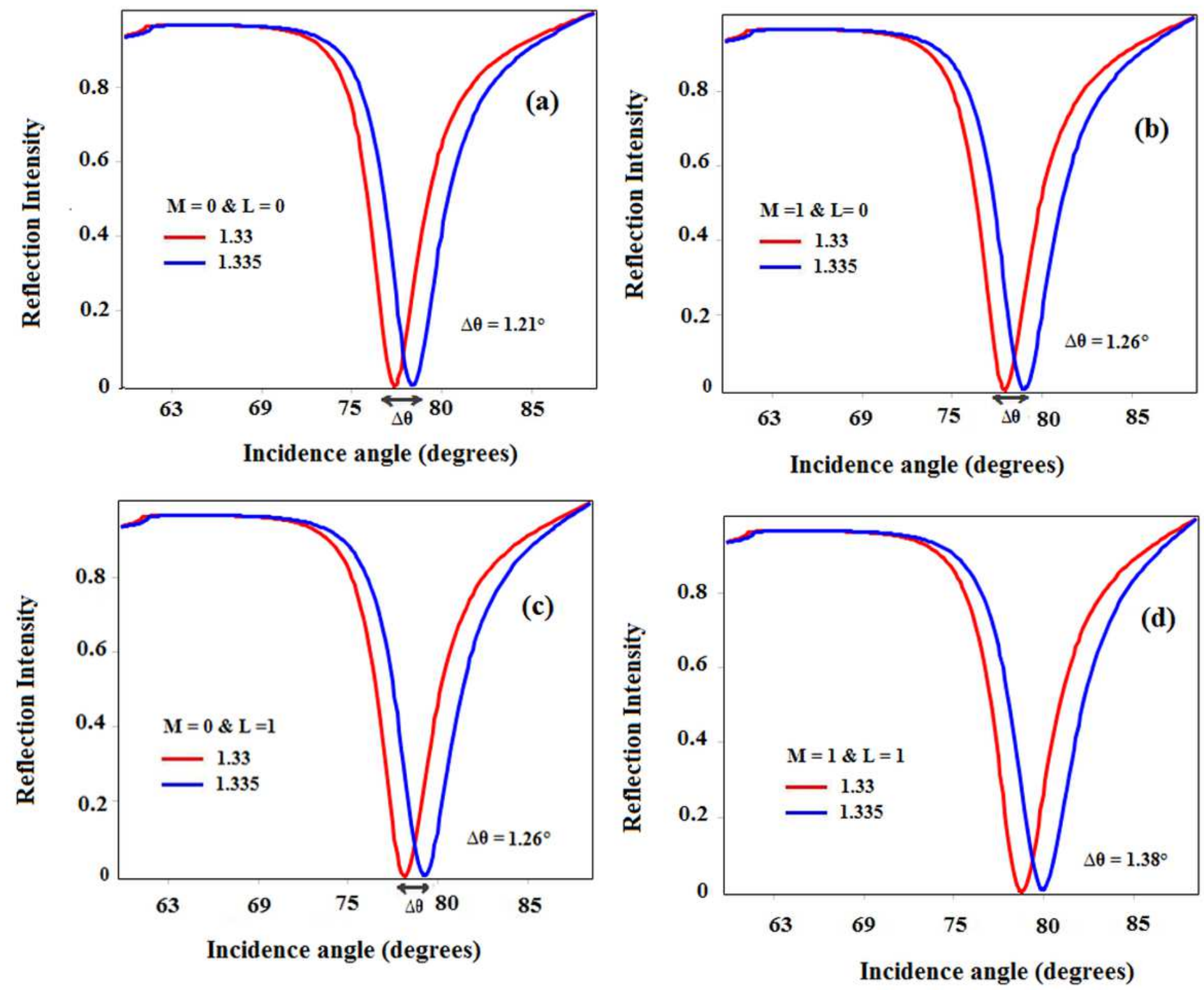

Figure 2

Reflection intensity as functions of incident angle with (a) $M=0$ and $L=0$, (b) $M=1$ and $L=0$, (c) $M=0$ and $L=1$ and (d) $M=1$ and $L=1$. 

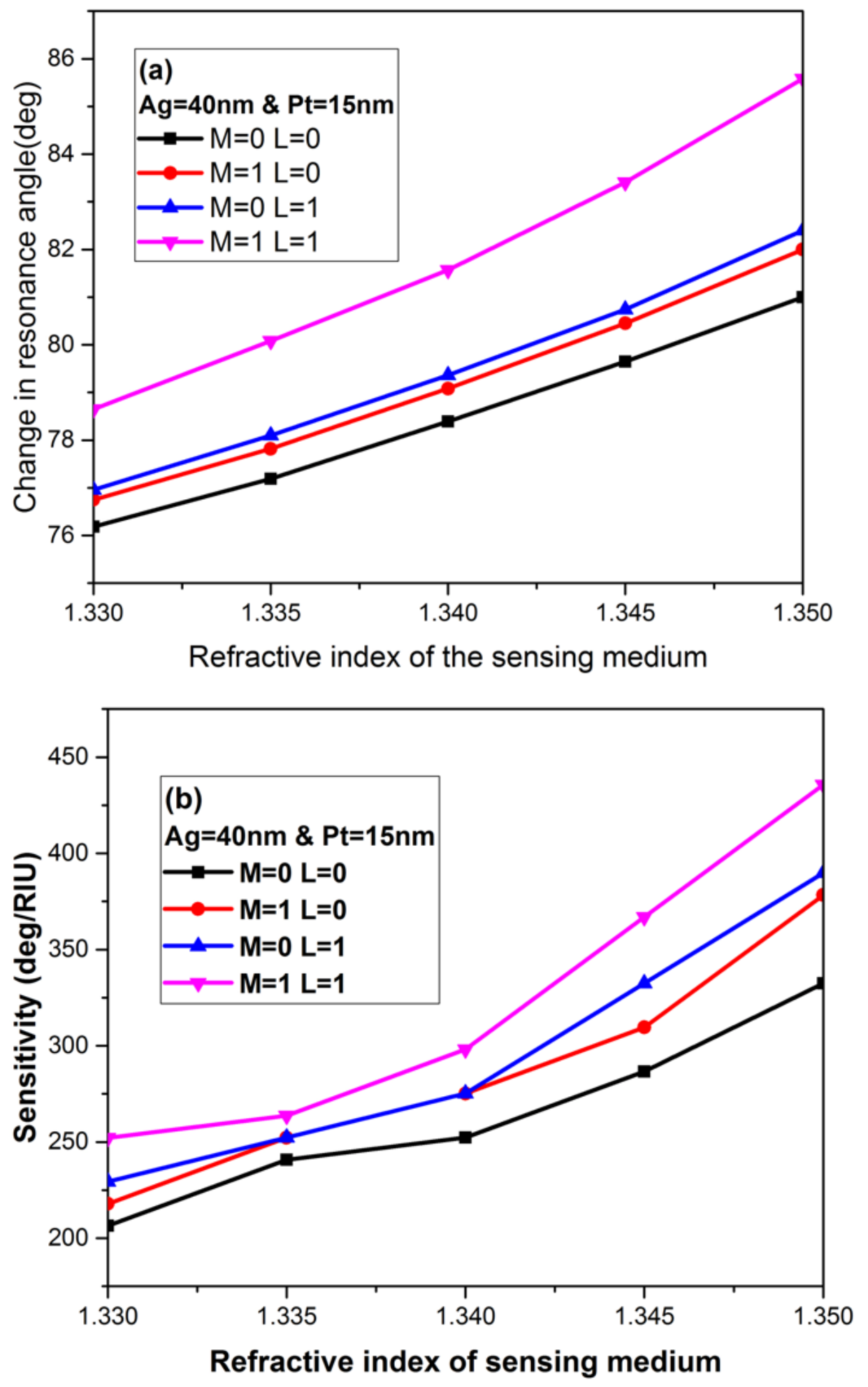

Figure 3

(a)Change in Resonance shift (b) Sensitivity corresponding to the R.I of the sensing medium from 1.33 to 1.335 . 

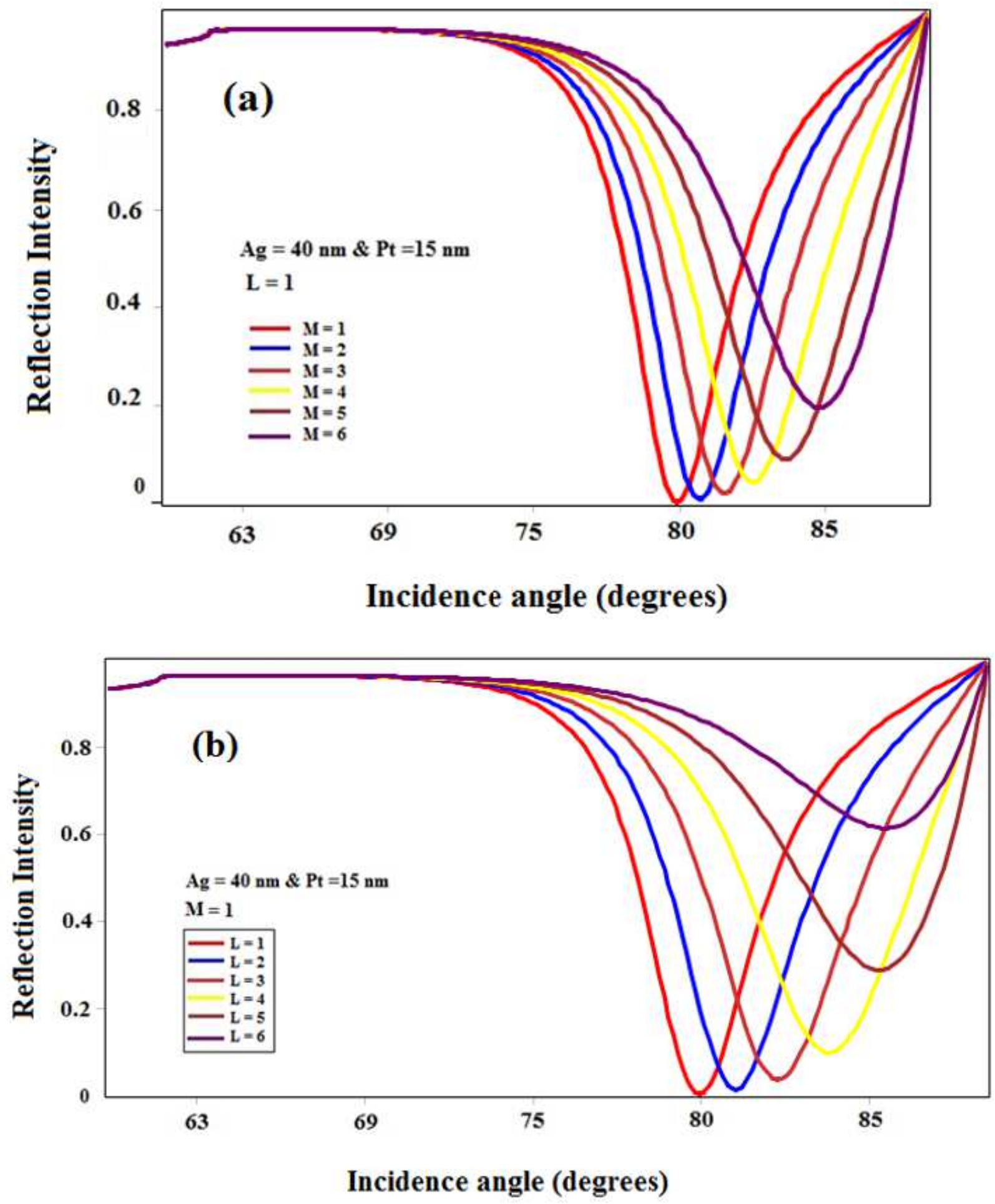

Figure 4

Reflection intensity as the function of incident angle (a) different layer of Black Phosphorus(BP) with mono layer of graphene (b) different layer of graphene with mono layer of Black Phosphorus(BP) 


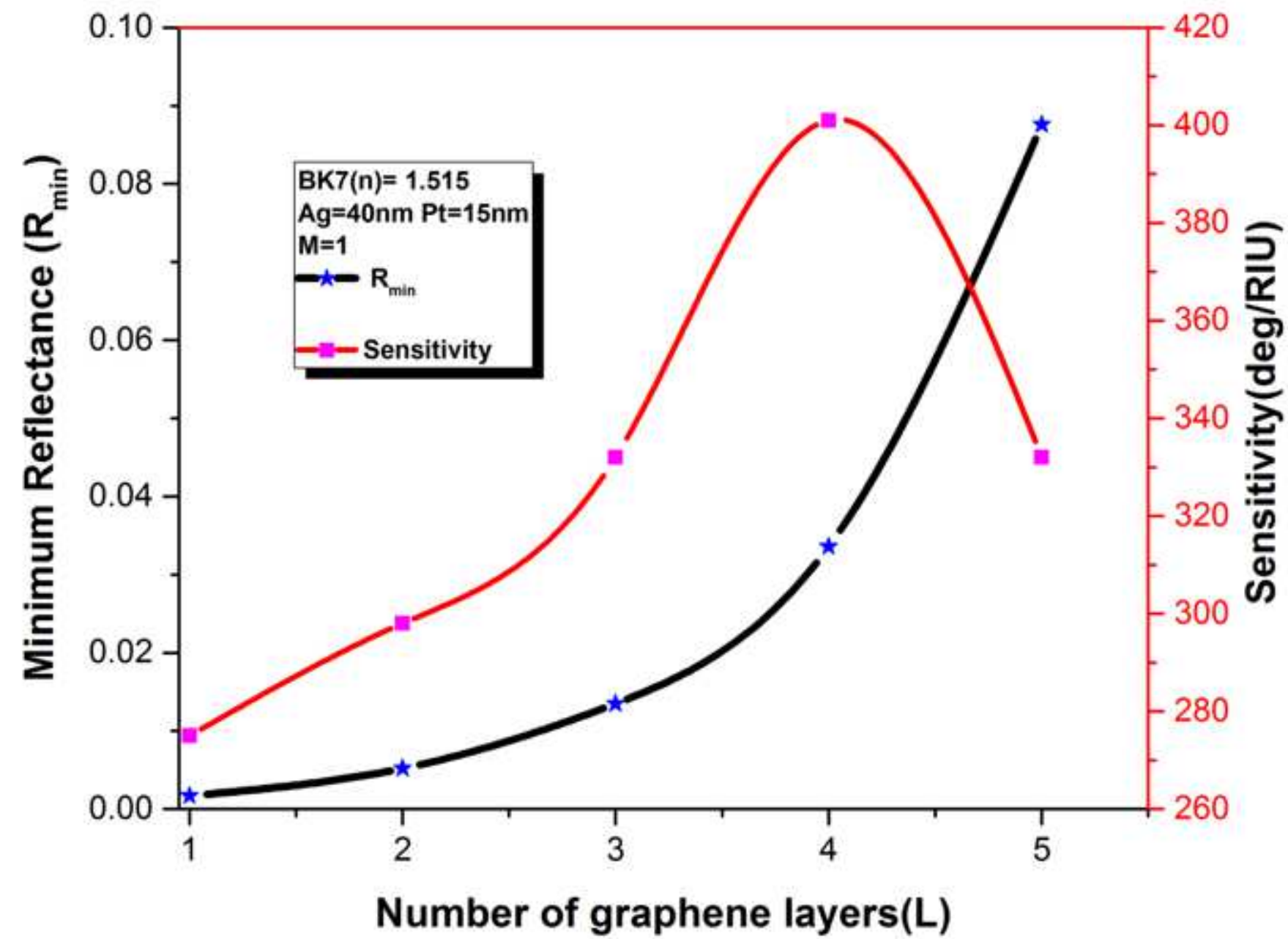

Figure 5

Minimum reflectance and Sensitivity as a functions of number of Graphene layers $(\mathrm{L})$ 


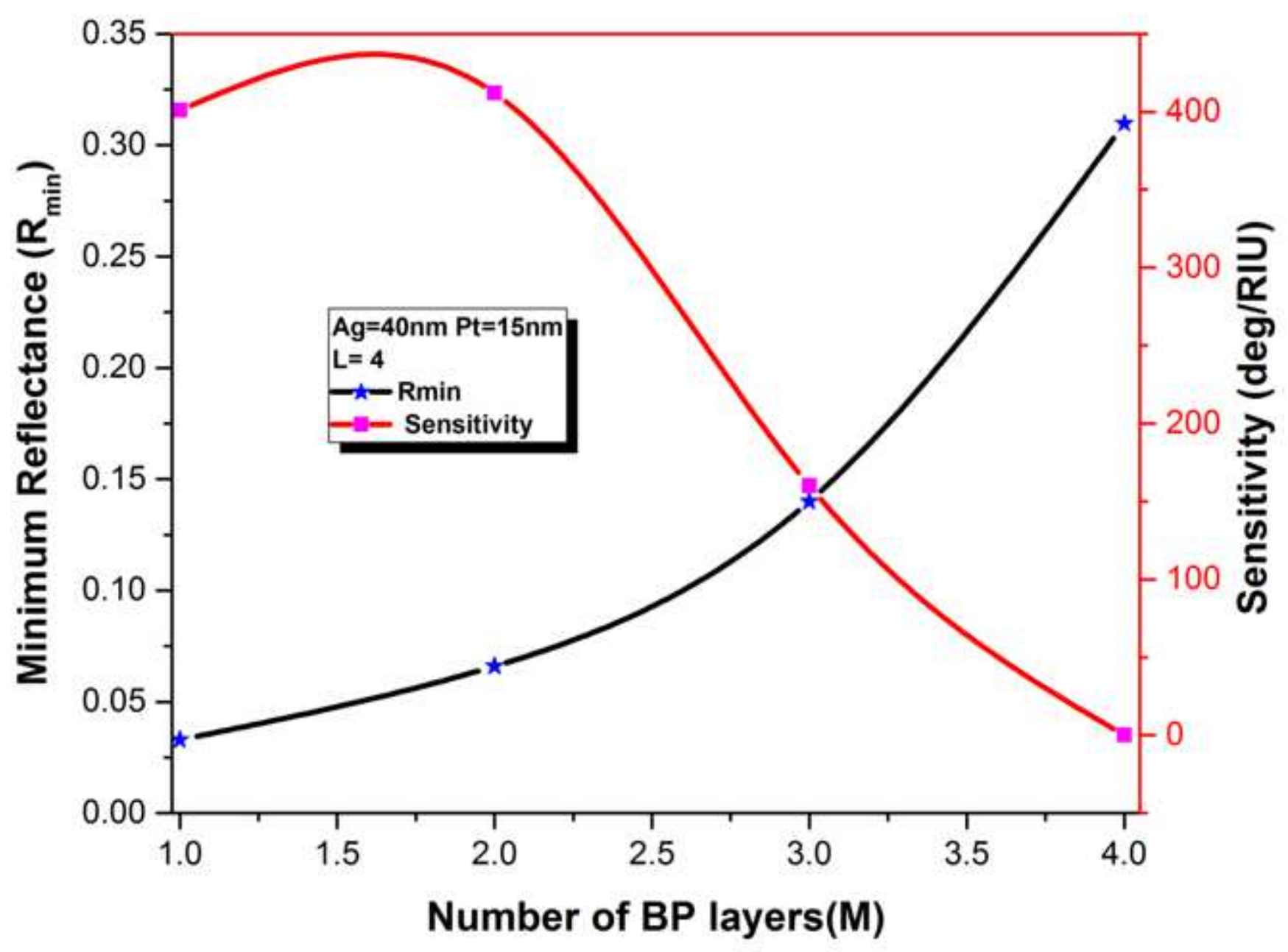

Figure 6

Minimum reflectance and Sensitivity as a functions of number of BP Layers(M) 


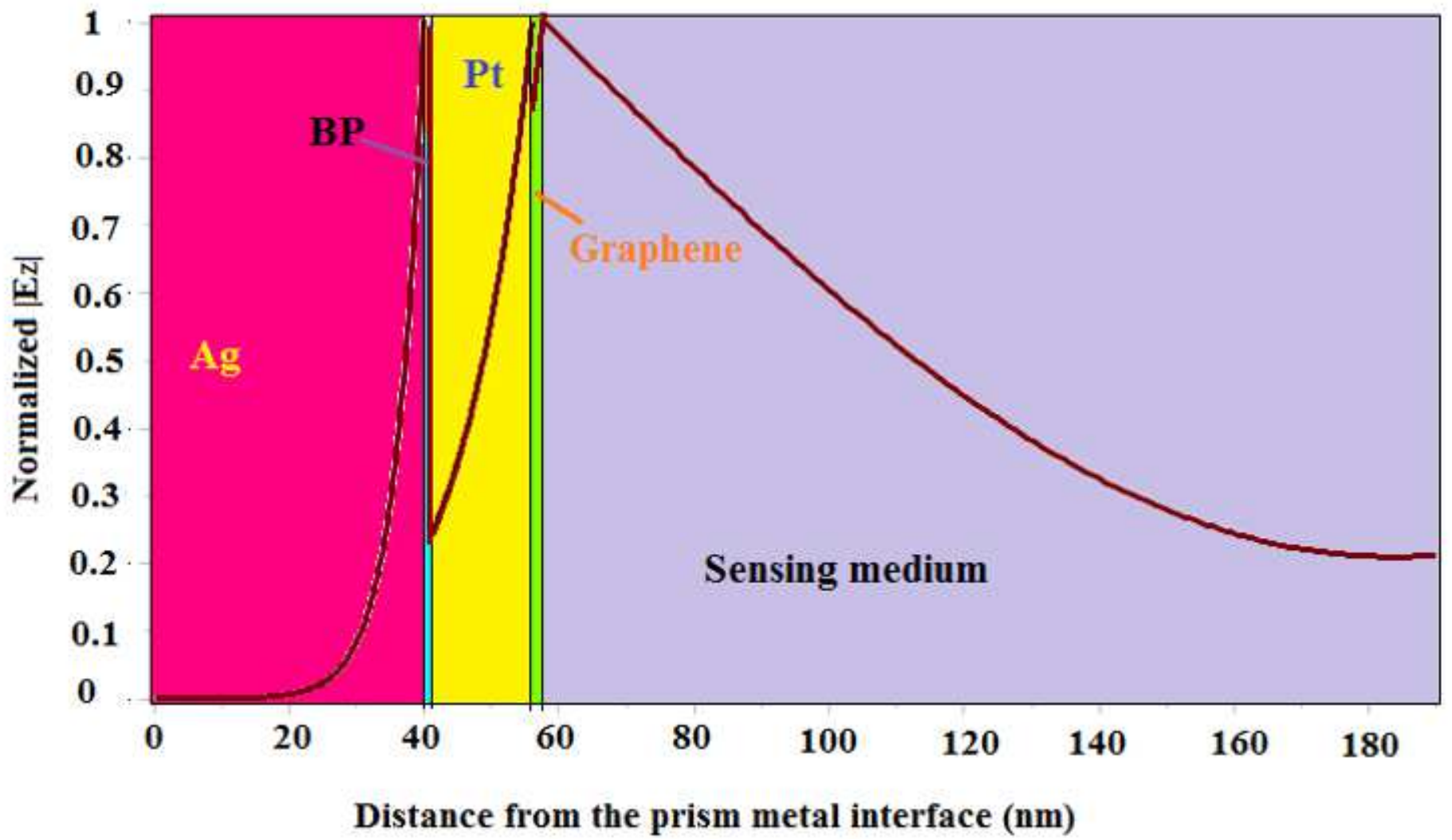

Figure 7

Normalized transverse magnetic electric field intensity versus distance normal from prism metal interface 